\title{
1 First genome-wide association study of non-severe malaria in two birth cohorts
}

\section{2 in Benin}

3 Jacqueline Milet $^{1^{*}}$, Anne Boland ${ }^{2 *}$, Pierre Luisi ${ }^{3,4}$, Audrey Sabbagh ${ }^{1}$, Ibrahim Sadissou ${ }^{5}$, Paulin

4 Sonon $^{5}$, Nadia Domingo ${ }^{6}$, Friso Palstra ${ }^{1}$, Laure Gineau ${ }^{1}$, David Courtin ${ }^{1}$, Achille Massougbodji ${ }^{6}$, 5 André Garcia ${ }^{1,6^{* *}}$, Jean-François Deleuze ${ }^{2 * *}$, Hervé Perdry ${ }^{7 * *}$.

$6 \quad{ }^{1}$ MERIT, IRD, Université Paris 5, Sorbonne Paris Cité, Paris, 75006, France; ${ }^{2}$ Centre National de

7 Recherche en Génomique Humaine (CNRGH), Institut de Biologie François Jacob, CEA, Université

8 Paris-Saclay, F-91057, Evry, France ; ${ }^{3}$ Centro de Investigación y Desarrollo en Inmunología y

9 Enfermedades Infecciosas, Consejo Nacional de Investigaciones Científicas y Técnicas, Cordoba,

10 Argentina ; ${ }^{4}$ Facultad de Filosofía y Humanidades, Universidad Nacional de Córdoba, Argentina ;

$11{ }^{5}$ Faculty of Medicine of Ribeirão Preto, University of São Paulo, Brazil; ${ }^{6}$ Centre d'Etude et de

12 Recherche sur le Paludisme Associé à la Grossesse et l'Enfance, Faculté des Sciences de la Santé,

13 Cotonou, Bénin ; ${ }^{7}$ Université Paris-Saclay, Centre de recherche en Epidémiologie et Santé des

14 Populations, Institut National de la Santé et de la Recherche Médicale, Villejuif, France

$15 *$ Joint first authors

$16 * *$ Joint last authors

17 The authors have no competing financial interests. 


\section{Abstract}

Recent research efforts to identify genes involved in malaria susceptibility using genome-wide approaches have focused on severe malaria. Here we present the first GWAS on non-severe malaria designed to identify genetic variants involved in innate immunity or innate resistance mechanisms. Our study was performed on two cohorts of infants from southern Benin (525 and 250 individuals respectively) closely followed from birth to 18-24 months of age, with an assessment of a space- and time-dependent environmental risk of exposure. Both the recurrence of mild malaria attacks and the recurrence of malaria infections as a whole (symptomatic and asymptomatic) were considered. Our study highlights a role of PTPRT, a tyrosine phosphatase receptor involved in STAT3 pathway and several other genes whose biological functions are relevant in malaria infection. Results shows that GWAS on non-severe malaria can successfully identify new candidate genes and inform physiological mechanisms underlying natural protection against malaria.

\section{Introduction}

In spite of numerous prevention and control efforts in recent years, malaria remains a major global public health problem with 219 million cases and 435,000 deaths in 2017 (1). In Africa, Bhatt et al. (2) have estimated that Plasmodium falciparum infection prevalence halved between 2000 and 2015, and that the incidence of clinical disease fell by 40\%, owing mainly to the large distribution of insecticide-treated nets, the most widespread intervention. However the fight against malaria is currently facing numerous challenges. A decrease in the global reduction of malaria cases and deaths is observed at present, with no significant progress made over the 2015-2017 period. The WHO African region which represents the region with the highest malaria burden (92\% of malaria cases and 93\% of malaria-related deaths in 2017) is also the area where medical advances have been hardest to achieve recently. Moreover control efforts are threatened by insecticide resistance and the possible spread of resistance to artemisinin (an essential component of the most efficient antimalaria drugs, the artemisinin-based combination therapy -ACT) from Southeast Asia to Africa. In 
absence of alternative treatments with the same efficacy and tolerability as ACT and of an efficient vaccine, fundamental malaria research continues to be essential in order to better understand the physiopathology of the disease.

$P$. falciparum is the most prevalent malaria parasite in sub-Saharan Africa (99.7\% of malaria cases in African region). Different clinical presentations of $P$. falciparum malaria exist, from asymptomatic (parasite carriage without any clinical sign), or mild forms (parasitemia with fever), to severe forms which may ultimately lead to death. This variability of clinical presentation is thought to be attributable to environmental factors, as well as parasite and human host factors, among which genetic variation could play a major role (3,4). In 2005, Mackinnon et al. (5) estimated that $24 \%$ and $25 \%$ of the variability in the incidence of mild malaria and severe malaria, respectively, could be explained by genetic factors. Numerous genetic epidemiological studies have attempted to identify gene polymorphisms associated with susceptibility or resistance to different malaria phenotypes (see $(4,6,7))$.

Most studies performed to date have focused on severe malaria, despite the fact that mild forms represent the major part of the global burden. Candidate gene studies for mild forms focused on genetic polymorphisms involved in host immune response (IL10, IL3, LTA,), in genes possibly involved in oxidative stress (HP), red blood cell (RBC) invasion by parasites (CR1, GRK5) or RBC defects. Among those, sickle cell trait (haemoglobin $\mathrm{S}, \mathrm{HbS}$ ), haemoglobin $\mathrm{C}(\mathrm{HbC})$ at homozygote state, alpha+ thalassemia and glucose-6-phosphate dehydrogenase (G6PD) deficiency have been associated with a protection against parasite invasion and clinical malaria attacks (7).

Other association studies have focused on chromosomal regions linked with parasite infection levels and mild malaria susceptibility, in particular 5q31-33 and 6p21-23 (8-15). In this last region, TNF has been the most studied candidate; NCR3, encoding a cell membrane receptor of natural killer, has been also repeatedly associated with mild malaria $(10,16)$.Since 2010, several genome-wide association studies (GWAS) and meta-analysis have been published on severe malaria (17-21). 
These studies confirmed the involvement of previously known susceptibility genes ( $H B B$ and $A B O)$ and revealed new genes (ATP2B4, the cluster of genes GYP/FREM3, among which GYPA and GYPB appear to play a central role (21)).

Here we present the first GWAS performed on mild malaria susceptibility. It was conducted on two cohorts of infants in southern Benin, used as discovery and replication cohorts (525 and 250 individuals respectively) and genotyped with the high density Illumina ${ }^{\circledR}$ HumanOmni5 beadchip. This study intended to identify genetic factors that play an early role in disease development (Figure 1), in cohorts of infants followed from birth to 18-24 months of age, thus targeting factors involved in innate immunity or innate resistance mechanisms.

In our study, infants were closely followed with symptomatic and asymptomatic malaria infections recorded. Furthermore geographical, climatic, behavioral and entomological data were collected in concomitant environmental risk surveys throughout the follow-up, to estimate a spatial- and timedependent risk of exposure. Association studies were performed with the recurrence of mild malaria attacks (RMM) and the recurrence of malaria infections (RMI) including mild malaria attacks and asymptomatic infections, taking into account a time-dependent risk of exposure. We find convincing evidence supporting the involvement of PTPRT, MYLK4, VENTX and UROC1 as well as strong association signals in PTPRM, ACER3 and CSMD1 all of which are relevant putative candidate genes in malaria physiopathology. 
86

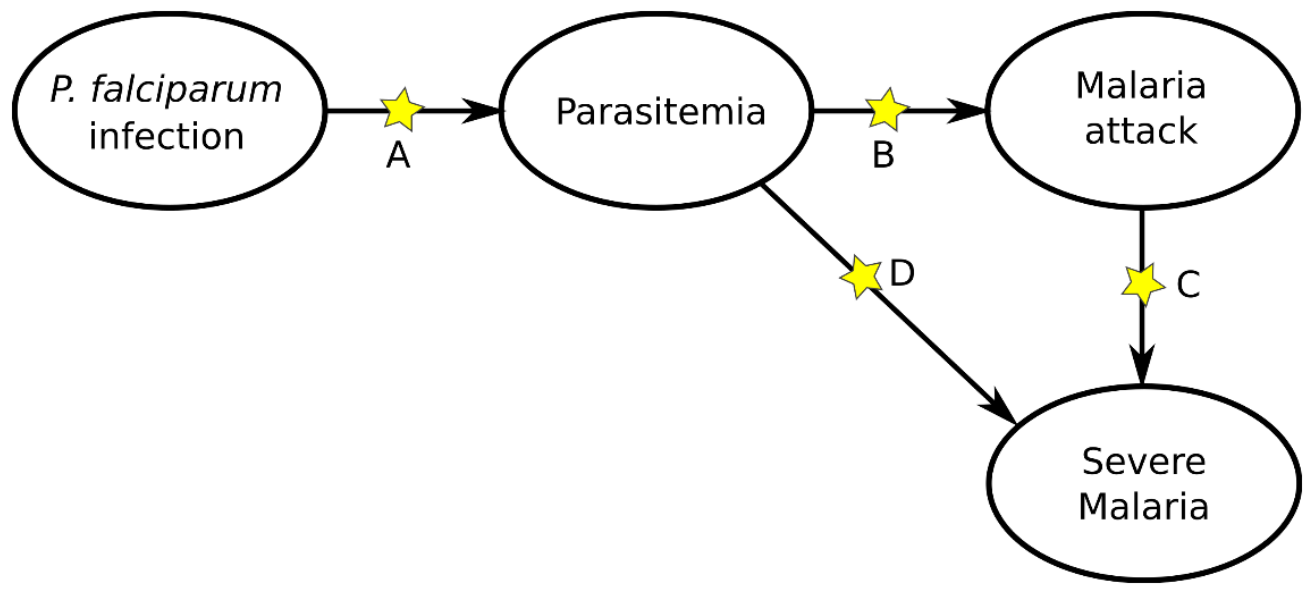

Figure 1. The path from $P$. falciparum infection to severe malaria, through parasitemia and malaria attacks (parasitemia with fever). The process may stop at each transition A, B, C and D, depending on several factors including the genetics of the host. Studies on severe malaria mainly target factors involved in transitions $\mathrm{C}$ and $\mathrm{D}$, while studies on mild malaria focus on $\mathrm{A}$ and $\mathrm{B}$. Our study focuses on transitions A and B in a population of infants.

\section{Results}

\section{Phenotypic and genotypic data}

Infants from both discovery (Tori-Bossito) and replication (Allada) cohorts come from southern Benin, from two sites located $20 \mathrm{~km}$ from each other, 40-50 km north-west of Cotonou, the economic capital. Ethnicity composition, based on self-reported ethnicity of the mother, significantly differed between the two study sites. The main ethnic groups were Tori (74\%) and Fon (11\%) in the first cohort whereas they were Aïzo (68\%) and Fon (22\%) in the second one. Each of the other ethnic groups accounted for less than $10 \%$ of individuals in each cohort.

Principal Component Analysis (PCA) performed on the two samples did not reveal any ethnic outliers and showed a low degree of stratification (Figure S1). PC1 separated the two cohorts and PC2 likely reflected some heterogeneity in the Tori-Bossito cohort. We observed that infants did not cluster by reported ethnicity, but rather by cohort and health center, indicating that the self-reported 
ethnicity of the mother is not a major factor of genetic heterogeneity in our samples. The PCA

including 1000 Genomes Project (KGP) populations (as described in methods) confirmed that our two cohorts are homogeneous (Figure S2). As expected, our two populations overlapped and clustered with Nigerian populations (Yoruba and Esan).

Main characteristics of infants were compared between samples included in or excluded from ethnic groups $(\mathrm{p}<0.04)$. For the replication cohort, only infants followed between 12 and 24 months of age were included in the GWAS. Thus a higher proportion of infants were excluded from school $(\mathrm{p}<0.01$ and $\mathrm{p}=0.06$ respectively).

For the 525 infants included in the discovery sample, mean (SD) length of follow-up was 16.9 months (2.83). A total of 342 infants (65.1\%) experienced at least one mild malaria attack (from one to 10 episodes) and 359 infants (68.4\%) were observed with at least one malaria infection (range

119 from one to 16 malaria infections by infant). For the replication cohort of 250 infants, mean (SD)

120 length of follow-up was 11.9 months (1.72). A proportion of 83.2\% and 86.8\% children experienced 121 at least one malaria attack (range 1 to 9), or at least one malaria infection (range 1 to 14) during the 122 follow-up, respectively.

\section{Adjusting on main epidemiological and environmental determinants}

124 Genome-wide association analyses were performed in two steps because computational burden

125 inherent to the random effect Cox model (23) makes it inappropriate for the large number of tests in 126 GWAS.

127 In the first step, the recurrence rate of malaria episodes was modeled using a random effect Cox 
model for recurrent events, taking into account epidemiological and environmental factors that might influence malaria infections in infants. A few covariates among all those considered were retained in the final model (Tables S3-S4). In each cohort, the same covariates were found associated for the recurrence of mild malaria attacks (RMM) and the recurrence of malaria infections (RMI). The levels of exposure to vector bites, health centers and transmission seasons were associated with the risk of infection in both cohorts. In addition, for the Allada cohort, significant effects were observed for marital status and for maternal education level. No effects of placental infection (detected by a thick and thin placental smears), low birth weight ( $<2500 \mathrm{~g}$ ) or HbS allele carriage were detected.

\section{Genome-wide association analyses}

After imputation using the KGP reference panel (phase 3), 15,566,900 high quality $\left(\mathrm{R}^{2}>0.8\right.$ ) correspond to individual effects which are not explained by the epidemiological covariates. The genomic inflation factor was consistent with a reliable adjustment for cryptic relatedness in our samples, for both analyses (Figure 2).

The analysis revealed four association signals with a $p$-value very close to the genome-wide

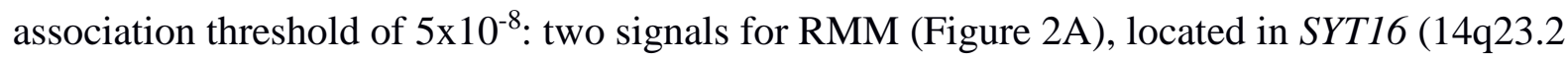
region, lead SNP rs375961263, $\mathrm{p}=3.7 \times 10^{-8}$ ) and in $P T P R M$, a gene encoding a receptor-type protein tyrosine phosphatase (18p11.23 region, lead SNP rs113776891, $\left.\mathrm{p}=3.77 \mathrm{x} 10^{-8}\right)$; and two

149 signals for RMI (Figure 2B): one located in ACER3, a gene encoding an alkaline ceramidase 150 (11q13.5 region, lead SNP rs77147099, $\mathrm{p}=6.85 \times 10^{-8}$ ) and another one located in PTPRT, a gene encoding a second receptor-type protein tyrosine phosphatase (20q12 region, lead SNP rs111968843, $\left.\mathrm{p}=9.70 \times 10^{-8}\right)$. 
A
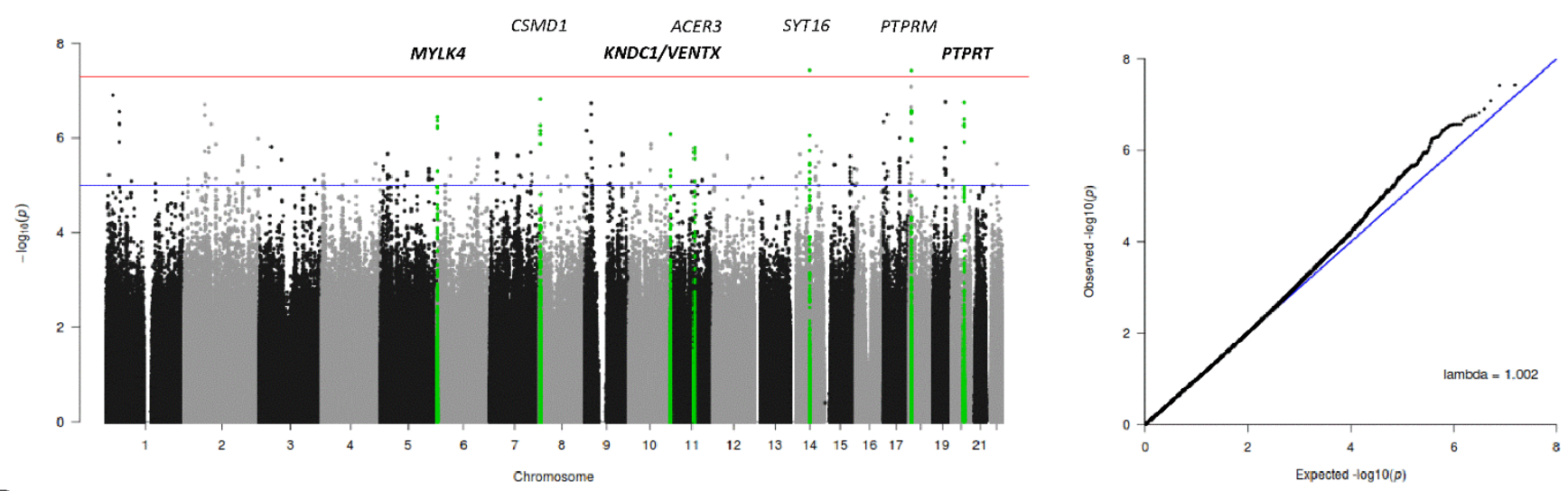

B
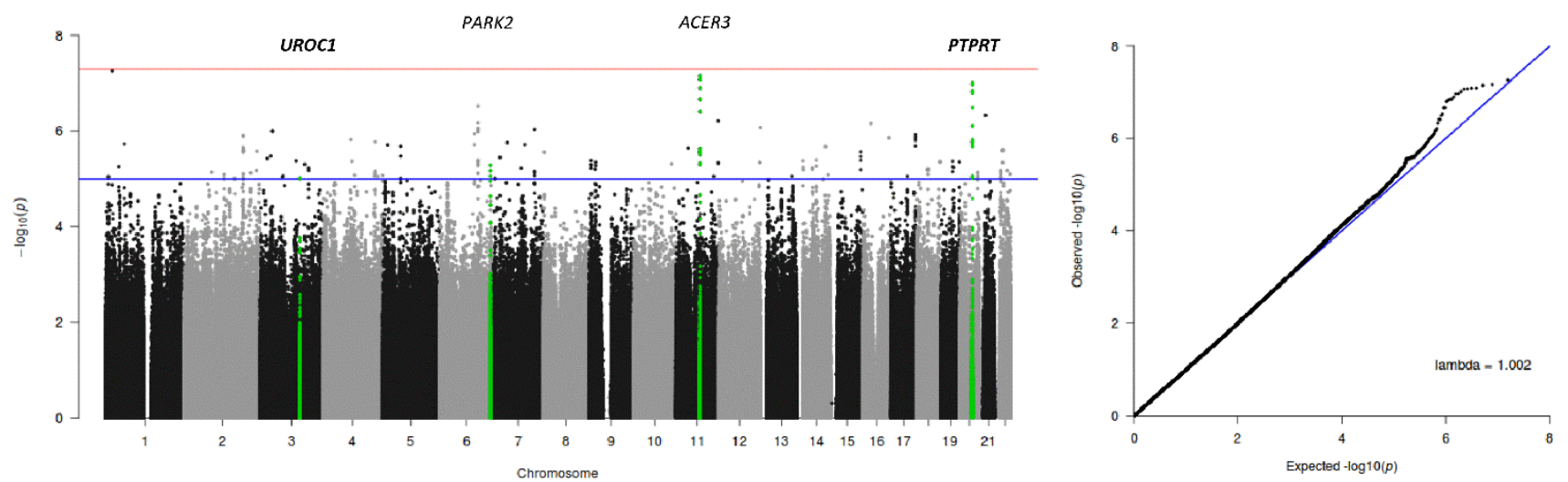

Figure 2. Manhattan plots and QQ plots for the discovery association study. A) GWAS results

infections. The red line in the Manhattan plots indicates the threshold of significance $\left(5 \times 10^{-8}\right)$, the blue line the threshold of suggestive association used for replication $\left(1 \times 10^{-5}\right)$. The blue line in the QQ plots shows the 1:1 regression line (the expected distribution of p-value under the null hypothesis). Lambda is the genomic inflation factor calculated as the ratio of the median of the empirically observed distribution of the test statistic to the expected median.

161 For these signals, data were re-analyzed in a single step with a mixed Cox model for recurrent events accounting for cryptic relatedness and relevant covariates, without resorting to intermediate confounder-adjusted phenotypes. Minor differences were observed in p-values and none of them reached genome-wide significance. 
A total of 356 and 214 variants were associated at $p<1 \times 10^{-5}$ with RMM and RMI respectively (Tables S5-S6), and were tested for replication in the second cohort. SNPs that showed evidence of replication $(p<0.05)$ are presented in Tables 1 and 2 . Highest statistical support was observed for SNPs located in the 6p25.2 locus with RMM (3 SNPs, min- $p=0.0056$ ). The most strongly associated SNP in the replication cohort, rs72840075, is located in an intron of MYLK4 which encodes a myosin light chain kinase (Figure 3). A meta-analysis of these SNPs showed a borderline significant association for SNP rs142480106 ( $\left.p=5.29 \times 10^{-8}\right)$ (Table 1).

A

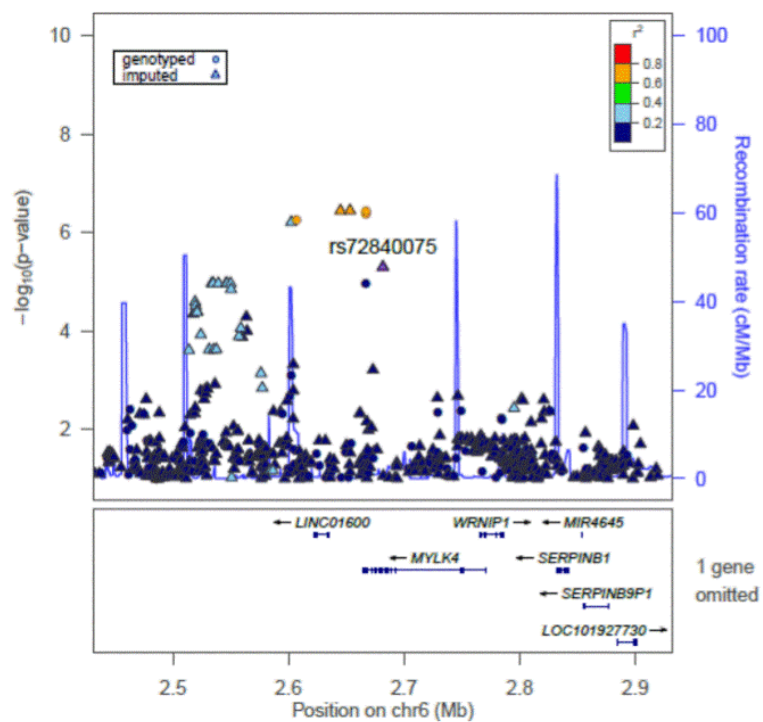

B

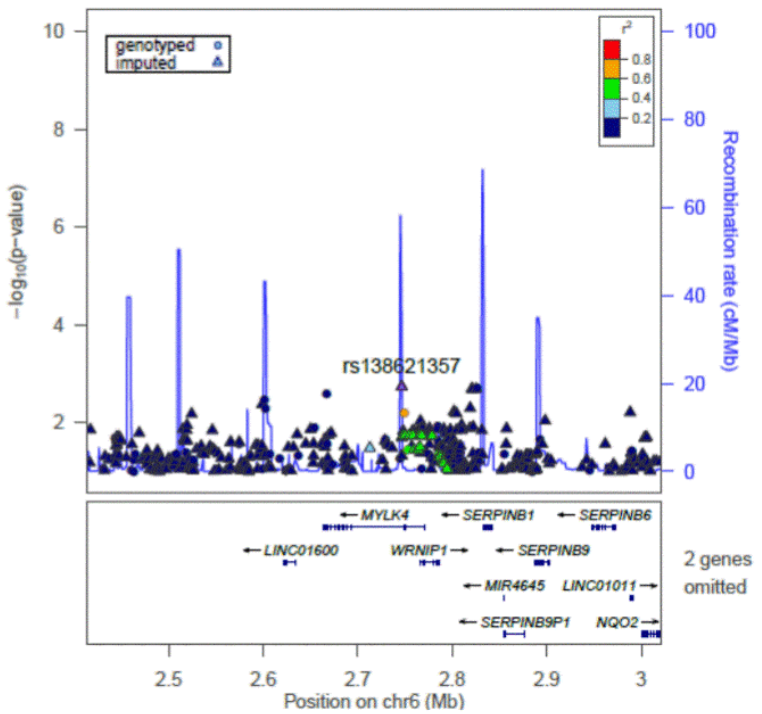

Figure 3. Annotated regional association plot for the 6p25.2 locus: A) Association results with populations) in the $250 \mathrm{~kb}$ region. 


\begin{tabular}{|c|c|c|c|c|c|c|c|c|c|c|}
\hline Locus & SNP & $\begin{array}{l}\text { Risk } \\
\text { allele }\end{array}$ & MAF & $\begin{array}{c}\underset{p}{\text { initial }} \\
\text { GWA }^{\mathrm{a}}\end{array}$ & $\begin{array}{c}p \\
\text { Cox model } \\
\text { Tori-bossito }^{\mathrm{b}}\end{array}$ & $\begin{array}{c}\text { HR (CI 95\%) } \\
\text { Tori-Bossito }\end{array}$ & $\begin{array}{c}p \\
\text { Cox model } \\
\text { Alladab }\end{array}$ & $\begin{array}{c}\text { HR (CI 95\%) } \\
\text { Allada }\end{array}$ & Nearest Genes & $\begin{array}{c}p \\
\text { Meta-analysis }\end{array}$ \\
\hline \multirow[t]{3}{*}{$6 \mathrm{p} 25.2$} & rs76088706 & $\mathrm{T}$ & 0.025 & $5.56 \times 10^{-7}$ & $2.68 \times 10^{-6}$ & $2.22(1.59-3.11)$ & 0.036 & $1.75(1.03-2.96)$ & C6orf195(Intergenic) & $3.65 \times 10^{-7}$ \\
\hline & rs142480106 & $\mathrm{T}$ & 0.019 & $4.35 \times 10^{-7}$ & $1.14 \times 10^{-6}$ & $2.52(1.73-3.66)$ & 0.013 & $2.08(1.17-3.70)$ & MYLK4 (UTR3) & $5.29 \times 10^{-8}$ \\
\hline & rs72840075 & A & 0.030 & $5.04 \times 10^{-6}$ & $1.05 \times 10^{-5}$ & $2.03(1.48-2.79)$ & 0.0056 & $1.88(1.20-2.95)$ & MYLK4 (intronic) & $2.01 \times 10^{-7}$ \\
\hline 10q26.3 & rs182416945 & A & 0.12 & $8.31 \times 10^{-7}$ & $5.56 \times 10^{-6}$ & $1.55(1.28-1.87)$ & 0.02 & $1.28(1.04-1.58)$ & KNDC1 (intronic) & $7.94 \times 10^{-7}$ \\
\hline 20q12 & rs6124419 & A & 0.12 & $1.21 \times 10^{-6}$ & $2.51 \times 10^{-6}$ & 1.53 (1.28-1.83) & 0.04 & $1.26(1.01-1.58)$ & $P T P R T$ (intronic) & $6.82 \times 10^{-7}$ \\
\hline
\end{tabular}

173 HR, hazard ratio; MAF, minor allele frequency

$174{ }^{a}$ p-value in GWA analysis performed in two steps using a confounder-adjusted phenotype.

$175{ }^{b}$ p-value of the association calculated with a random effect Cox model accounting for cryptic relatedness and relevant covariates (health center, risk of exposure, transmission season for both cohorts, marital status and education of women in addition for Allada cohort)

\section{Table 2. Association signals which replicated at $\mathbf{p}<0.05$ for the recurrence of malaria infections.}

\begin{tabular}{|c|c|c|c|c|c|c|c|c|c|c|}
\hline Locus & SNP & $\begin{array}{l}\text { Risk } \\
\text { allele }\end{array}$ & MAF & $\underset{\text { initial GWA }^{\mathrm{a}}}{p}$ & $\begin{array}{c}p \\
\text { Cox model } \\
\text { Tori-bossito }\end{array}$ & $\begin{array}{c}\text { HR (CI 95\%) } \\
\text { Tori-Bossito }\end{array}$ & $\begin{array}{c}p \\
\text { Cox model } \\
\text { Alladab }^{\mathbf{b}}\end{array}$ & $\begin{array}{l}\text { HR (95\%) } \\
\text { Allada }\end{array}$ & Nearest Genes & $\begin{array}{c}p \\
\text { Meta-analysis }\end{array}$ \\
\hline 3q21.3 & rs9871671 & $\mathrm{A}$ & 0.11 & $9.79 \times 10^{-6}$ & $5.00 \times 10^{-5}$ & $1.49(1.22-1.81)$ & 0.04 & $1.30(1.01-1.66)$ & UROC1 (exonic) & $8.25 \times 10^{-6}$ \\
\hline 20q12 & rs6124419 & A & 0.12 & $3.19 \times 10^{-7}$ & $1.35 \times 10^{-6}$ & $1.58(1.31-1.91)$ & 0.04 & $1.28(1.00-1.64)$ & PTPRT (intronic) & $4.10 \times 10^{-7}$ \\
\hline
\end{tabular}

179 HR, hazard ratio; MAF, minor allele frequency

$180{ }^{\mathrm{a}} \mathrm{p}$-value in GWA analysis performed in two steps using a the confounder-adjusted phenotype

181 b p-value of the association calculated with a random effect Cox model accounting for cryptic relatedness and relevant covariates (health center, risk of

182 exposure, transmission season for both cohorts, marital status and education of women in addition for Allada cohort) 
The same SNP, rs6124419, located in intron 18 of PTPRT, replicates for both phenotypes. The regional linkage disequilibrium (LD) plot showed a narrow signal of around $20 \mathrm{~kb}$ width in both cases which encompasses exons 20 to 22 (Figure 4A). An estimation of the incidence rate of mild malaria attacks in three risk groups based on exposition levels showed that the effect of rs6124419 is consistent regardless of the exposition level and the cohort (Figure 4C). Interestingly PTPRT is a paralogue of $P T P R M$, for which a highly suggestive association peak was observed with mild malaria attacks in the 18p11.23 chromosomal region (Figure 4A).

A

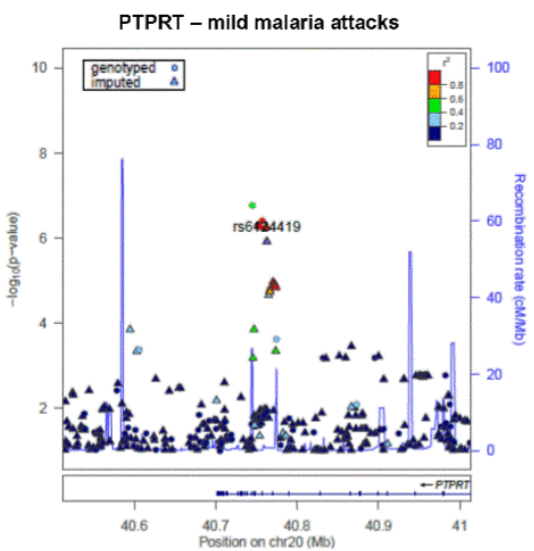

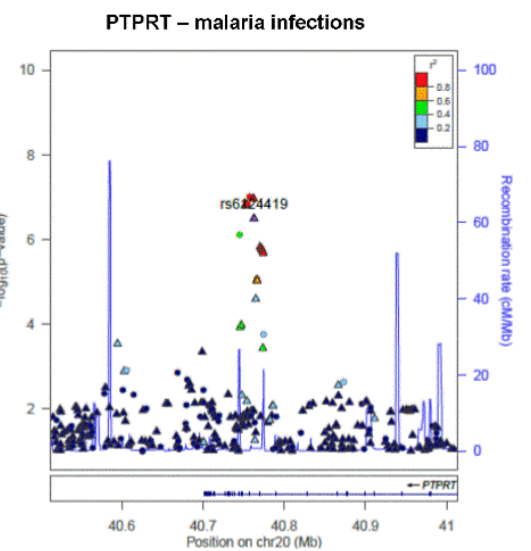

C

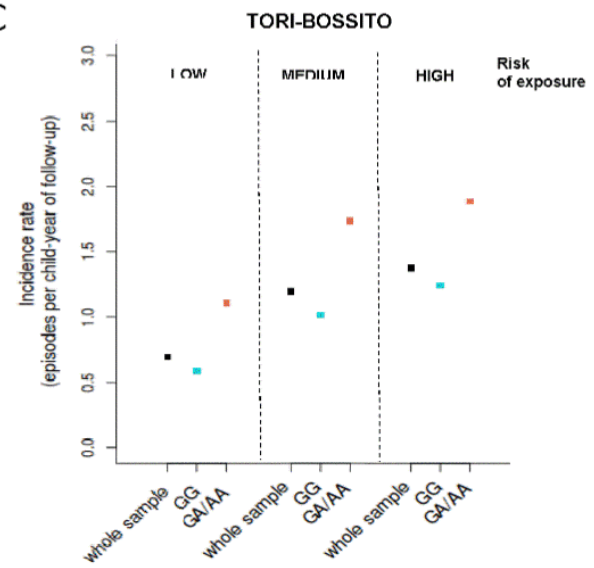

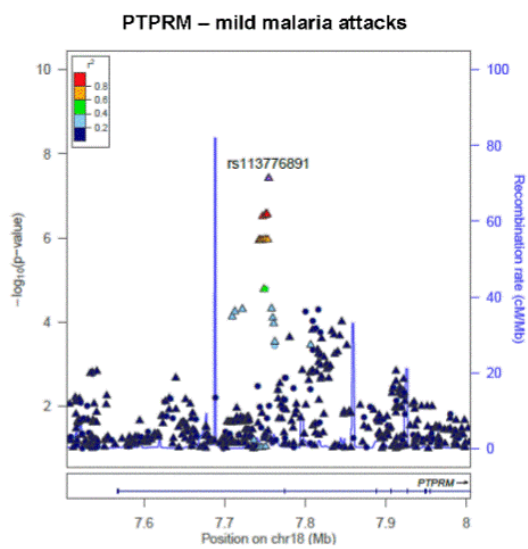

B

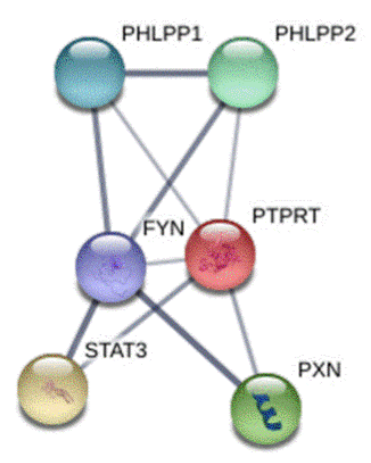

Figure 4. A) Annotated regional association plots for PTPRT and PTPRM loci. B) Interaction network for PTPRT C) Crude incidence rate of mild malaria attacks for rs6124419 genotypes calculated for three classes of environmental exposure. Plots show LD calculated on Nigerian Population of KGP dataset (ESN and YRI populations). Replicating SNP are highlighted for 
PTPRT. The three classes were defined from the mean environmental exposure risk across all the follow-up calculated for each infant. Low, medium and high correspond to the three tertiles of the distribution.

Other associations which replicated in the Allada cohort include rs182416945 in KNDC1 and rs9871671 in UROC1 (Table 2). This last one, a missense SNP, is predicted to be probably damaging with a score of 0.99 in PolyPhen-2(24) and has a Combined Annotation Dependent deleteriousness substitutions of the human genome. and a conditional analysis was run (Figure 4A and Figures S3 and S4). For none of them, a significant residual signal was found.

\section{Functional annotation and gene mapping of GWAS results}

To identify the genes and variants most likely associated with these four replicating association signals, and to select potential genes of interest pointed out by non-replicating signals, we used the FUMA web platform (27). FUMA identifies independent associated genomic regions and prioritizes genes based on functional consequences of SNPs in each of them.

Thirty independent associated regions were identified for RMM and 15 for RMI, based on the presence of at least 3 SNPs below the $p$-value threshold of $10^{-5}$ in a region (LD threshold to aggregate SNPs in one signal, $\mathrm{r}^{2}=0.1$ ). Candidate SNPs (among SNPs present either in our data or in the AFR population of the KGP reference panel) were then defined based on $L D\left(r^{2}>0.6\right)$ with the lead SNP in each of these regions, or with one of the replicating SNPs. These candidate SNPs were mapped to genes, by positional mapping (deleterious coding SNPs -

218 Regulome database (RDB, (28)) score $\leq 2$ ), and by eQTL mapping based on expression data from 219 relevant tissue types for malaria (whole blood, cell-EBV transformed lymphocytes, liver, skin, cells transformed fibroblasts and spleen). These mappings identified 20 genes for RMM and 8 for RMI 
221 (Tables S7-S8). Results for the strongest signals of association with both phenotypes are

summarized in Figure 5. We additionally performed chromatin interaction mapping with high chromosome contact map (Hi-C) data (29) with FUMA, allowing to identifying genes located in regions with significant chromatin interaction with the candidate SNPs (Figure 5).

For each of the four replicating loci FUMA identified a single gene, suggesting that these genes are very likely to be the "causal gene" in these regions. Three of these highlighted genes correspond to the gene in which the replicating SNP is located: MYLK4 in 6p25.2 region, PTPRT in 20q12 region, UROC1 in 3q21.3 region. The last highlighted gene, VENTX in 10q26.3 region, is located 17kb from the lead SNP. In MYLK4 locus, the replicating SNP, rs72840075, is an eQTL in whole blood (eQTLGen and BIOS eQTL (30) database, FDR<0.05). Allele at risk in our data is associated with a higher expression of this gene. One deleterious SNP is observed in PTPRT $\left(\mathrm{r}^{2}=0.78\right.$ with the replicating SNP, CADD = 14.39), and in UROC1 (the aforementioned deleterious SNP). In VENTX locus, two SNPs, rs182416945 (the replicating one) and rs138609386, in complete DL $\left(\mathrm{r}^{2}=1\right)$ with the first one, were identified as a significant eQTL in whole blood (eQTLGen database). The allele at risk in our data is associated with a lower expression of VENTX, a gene coding for the VENT Homeobox.

FUMA highlighted also PTPRM as the most probable gene associated with the prominent signal for RMM in 18p11.23 region. Two candidate SNPs, rs138057394 and rs112288193 $\left(\mathrm{r}^{2}=0.74\right.$ with the lead SNP, for both SNPs) are annotated as deleterious coding SNPs (CADD score of 19.58 and 14.64 respectively).

Furthermore, among the other genes pointed out by FUMA (Figure 5) in non-replicating signals, ACER3 in 11q35.5 region is identified by the three mapping methods performed. Numerous significant eQTL associations were observed in the cells transformed fibroblast tissue (GTEx v7 database). For these eQTL, alleles at risk in our data were associated with a lower expression of the gene. This association signal is the strongest signal for RMI, with a $p$-value approaching the genome-wide significance threshold, ( $p=6.85 \times 10^{-8}$, figure 2$)$ and it was also found associated 
with $\operatorname{RMM}\left(p=1.60 \times 10^{-6}\right)$.

\begin{tabular}{|c|c|c|c|c|c|c|c|c|c|c|c|c|c|c|}
\hline \multirow[b]{3}{*}{ Phenotype } & \multirow[b]{3}{*}{ Locus } & \multirow[b]{3}{*}{ Location (start - end) } & \multirow[b]{3}{*}{ Lead SNP } & \multirow[b]{3}{*}{ MAF } & \multirow[b]{3}{*}{ P intial GWAS } & \multirow[b]{3}{*}{ Gene } & \multicolumn{8}{|c|}{ functional consequences on gene } \\
\hline & & & & & & & \multirow[b]{2}{*}{$e^{\circ}$} & \multirow[b]{2}{*}{$\hat{\mathscr{\sigma}}$} & \multirow[b]{2}{*}{0} & \multicolumn{3}{|c|}{ positional mapping } & \multicolumn{2}{|c|}{ eQTL mapping } \\
\hline & & & & & & & & & & rsID & $\begin{array}{l}\text { CADD } \\
\text { score }\end{array}$ & $\begin{array}{l}\text { RDB } \\
\text { score }\end{array}$ & nSNPs & $\begin{array}{l}\text { eQTL } \\
\text { dir. }\end{array}$ \\
\hline \multirow{10}{*}{$\begin{array}{c}\text { Mild malaria attacks } \\
\text { RMM }\end{array}$} & $6 \mathrm{p} 25.2$ & $2602462-2681733$ & rs547331171 & 0.03 & $3.61 \times 10-7$ & MYLK4 & & & & & & & 1 & + \\
\hline & $10 \mathrm{q} 26.3$ & $135034267-135052777$ & rs182416945 & 0.12 & $8.31 \times 10-7$ & VENTX & & & & & & & 2 & - \\
\hline & $20 q 12$ & 40745108-40774357 & rs111968843 & 0.11 & $1.21 \times 10-6$ & PTPRT & & & & rs144104706 & 14.39 & & & \\
\hline & $18 p 11.23$ & 7742657-7754654 & rs113776891 & 0.08 & $3.78 \times 10-8$ & PTPRM & & & & $\begin{array}{l}\text { rs138057394 } \\
\text { rs112288193 }\end{array}$ & 19.58 & $2 b$ & & \\
\hline & 18p11.31 & 7012462-7034932 & rs143310084 & 0.02 & $8.32 \times 10-8$ & LAMA1 & & & & rs566655 & 14.64 & & & \\
\hline & $1 \mathrm{p} 34.2$ & $41902797-41932682$ & rs 75470436 & 0.01 & $2.76 \times 10-7$ & CTPS1 & & & & & & & 1 & + \\
\hline & $2 \mathrm{p} 12$ & $80290041-80298707$ & rs6708548 & 0.08 & $1.58 \times 10-6$ & CTNNA2 & & & & \begin{tabular}{|l|} 
rs1484465 \\
rs1484464
\end{tabular} & 19.08 & & & \\
\hline & $11 q 13.5$ & $76570145-76654016$ & rs77147099 & 0.02 & $1.60 \times 10-6$ & ACER3 & & & & & & & 7 & - \\
\hline & $5 p 14.2$ & $24307924-24461650$ & rs12519312 & 0.01 & $2.15 \times 10-6$ & C5orf17 & & & & & & & 11 & - \\
\hline & $5 p 14.3$ & 22318243-22620207 & rs 141690513 & 0.03 & $2.16 \times 10-6$ & $\mathrm{CDH} 12$ & & & & rs141690513 & 14.03 & & & \\
\hline \multirow{8}{*}{$\begin{array}{c}\text { Malaria infections } \\
\text { RMI }\end{array}$} & $20 q 12$ & $40745108-40774357$ & rs111968843 & 0.11 & $9.70 \times 10-8$ & PTPRT & & & & rs144104706 & 14.39 & & & \\
\hline & $3 q 21.3$ & $126218211-126218211$ & rs9871671 & 0.11 & $9.79 \times 10-6$ & UROC1 & & & & rs9871671 & 26.50 & & & \\
\hline & $11 q 13.5$ & 76418359-76739604 & rs77147099 & 0.02 & $6.85 \times 10-8$ & ACER3 & & & & \begin{tabular}{|l|} 
rs141181984 \\
rs11608202
\end{tabular} & 13.95 & $2 \mathrm{~b}$ & 21 & - \\
\hline & $6 q 22.31$ & 122845480-123163778 & rs146900853 & 0.11 & $3.00 \times 10-7$ & SMPDL3A & & & & & & & 1 & + \\
\hline & $11 q 25$ & $133411892-133432324$ & rs7929384 & 0.05 & $6.14 \times 10-7$ & OPCML & & & & $\begin{array}{l}\text { rs7126528 } \\
\text { rs7126348 }\end{array}$ & 17.04 & & & \\
\hline & $2 q 32.1$ & 188435603-188473204 & rs7583251 & 0.16 & $1.25 \times 10-6$ & TFPI & & & & rs 115976332 & & $2 \mathrm{~b}$ & & \\
\hline & $6 q 26$ & 161851951-162045282 & rs189683911 & 0.08 & $5.16 \times 10-6$ & PRKN & & & & rs80324971 & & $2 a$ & & \\
\hline & $20 q 13.32$ & $58143026-58143026$ & rs 144135811 & 0.15 & $6.83 \times 10-6$ & MED15 & & & & $\begin{array}{l}\text { rs } 114819925 \\
\text { rs5758468 }\end{array}$ & & $1 \mathrm{a}$ & 1 & - \\
\hline
\end{tabular}

Figure 5. Genes identified by FUMA based on functional consequences of SNPs, in

reported are those with at least one gene identified based on positional mapping or eQTL mapping.

was identified as the replicating SNP or the SNP with the lowest $p$-value. The $p$-value of the initial

254 GWA was calculated with a linear mixed model on the adjusted phenotypes. Filled red boxes

255 indicate whether the gene was identified by positional mapping (pos.), eQTL mapping (eQTL) or

256 chromatin interaction mapping (CI). For positional mapping, deleterious SNP names are given

257 (rsID) with the maximum CADD score and RegulomDB score observed for these SNPs. For eQTL

258 mapping, the number of significant eQTL associations (nSNPs) are reported, together with the

259 direction of effect allele to gene expression, for the risk increasing allele of GWAS (eQTL

260 direction). 


\section{Candidate associations}

263 We also tested the statistical significance of genes and variants previously reported in literature as

264 associated with malaria (Table 3). Only SNP rs40401 in IL3 was found marginally associated with

265 both phenotypes ( $\mathrm{p}=0.01$ and 0.02 respectively). Moreover, two suggestive peaks are observed in

266 the candidate regions: one in the IL3 locus with both phenotypes (10 SNPs encompassing IL3 and

267 exon 1 to 3 of $A C S L 6$, min-p $=8.89 \times 10^{-5}$ with RMM and min-p=1.66x10 ${ }^{-4}$ with RMI, rs7714191)

268 and one in the IL10 locus with RMM, $26 \mathrm{~kb}$ upstream of the gene (4 SNPs, min- $\mathrm{p}=2.04 \times 10^{-4}$, 269 rs116126622). 
Table 3. Candidate SNP associations

\begin{tabular}{|c|c|c|c|c|c|c|c|c|c|}
\hline \multirow{2}{*}{ Gene } & \multirow{2}{*}{ SNP } & \multirow{2}{*}{ Variant } & \multirow{2}{*}{$\begin{array}{l}\text { Location } \\
\text { (GRCh37) }\end{array}$} & \multirow{2}{*}{ MAF } & \multirow{2}{*}{ Change } & \multicolumn{2}{|c|}{ Malaria attacks } & \multicolumn{2}{|c|}{ Malaria infections } \\
\hline & & & & & & $\beta($ CI 95\%) & $\mathbf{p}$ & $\beta($ CI 95\%) & $\mathbf{p}$ \\
\hline \multirow[t]{2}{*}{ HBB } & rs334 & $\mathrm{HbS}$ & $11: 5248232$ & 0.10 & $\mathrm{~A}>\mathrm{T}$ & -0.040 & 0.24 & 0.05 & 0.34 \\
\hline & rs33930165 & $\mathrm{HbC}$ & 11:5248233 & 0.04 & $\mathrm{G}>\mathrm{A}$ & -0.011 & 0.83 & -0.01 & 0.95 \\
\hline \multirow[t]{2}{*}{$T N F$} & rs1799964 & 3’UTR, TNF -857 & $6: 31574531$ & 0.19 & $\mathrm{~T}>\mathrm{C}$ & 0.002 & 0.92 & 0.02 & 0.59 \\
\hline & rs1800629 & 3’UTR, TNF -308 & $6: 31543031$ & 0.11 & $\mathrm{G}>\mathrm{A}$ & 0.018 & 0.11 & -0.011 & 0.80 \\
\hline IL10 & rs1800871 & 3’UTR, IL10 -819 & $1: 206946634$ & 0.38 & $\mathrm{C}>\mathrm{T}$ & 0.023 & 0.23 & 0.02 & 0.51 \\
\hline IL3 & rs40401 & Exon 1, missense & $5: 131396478$ & 0.71 & $\mathrm{C}>\mathrm{T}$ & 0.050 & 0.01 & -0.08 & 0.02 \\
\hline \multirow[t]{2}{*}{$A B O$} & rs8176746 & Exon 10, missense & $9: 136131322$ & 0.15 & $C>A$ & -0.022 & 0.42 & -0.062 & 0.13 \\
\hline & rs8176719 & Exon 9, frameshift & 9:136132908 & 0.27 & $->G$ & -0.027 & 0.18 & -0.038 & 0.22 \\
\hline \multirow[t]{2}{*}{ ATP2B4 } & rs1541255 & Exon 1 & 1:203652141 & 0.41 & $A>G$ & -0.006 & 0.75 & -0.035 & 0.24 \\
\hline & rs10900585 & Intron 2 & 1:203654024 & 0.46 & $\mathrm{G}>\mathrm{T}$ & 0.000 & 0.96 & 0.014 & 0.65 \\
\hline
\end{tabular}

271 MAF, minor allele frequency;

272 Results of association tests performed in the discovery cohort using the confounder-adjusted phenotype. $\beta$ is the estimate of the SNP effect under an

273 additive model. A negative value indicates a protective effect of the alternative allele and conversely. 


\section{Discussion}

275 Genetic factors of resistance to malaria may be involved at different stages during the course of the disease: inoculation of parasite, development of asymptomatic parasitemia, appearance of clinical symptoms including fever, rapid progress to complications and manifestation of severe malaria (4). However research efforts to identify host genetic factors have so far mainly focused on severe malaria, the life threatening form of the disease. While some genetic factors may be in common between severe and mild forms, studies on severe malaria are not designed to discover genes affecting specifically the risk of malaria infection and the development of mild malaria. For this reason, studies based on non-severe malaria forms may help to complete our understanding of the disease.

Our study represents the first screening of genetic variations associated with non-severe forms of malaria at a genome-wide level. It was conducted on two birth cohorts closely followed with a protocol of surveillance which allowed to ascertain malaria infections as a whole. Infants were visited at home weekly or bi-monthly during all the follow-up for the systematic detection of fever and symptomatic malaria. Moreover, a TBS was performed monthly to detect asymptomatic infections $(23,24)$. Another strength of our study is the assessment of environmental risk of exposure at an individual level all along the follow-up. Although there is consistent evidence for local variation in exposure to $P$. falciparum, which may partly explain the heterogeneity in malaria infection incidence observed in children $(31,32)$, this factor has been seldom considered in genetic epidemiology studies. Here, environmental risk of exposure was taken into account in an unprecedented manner. Entomological, climatic, environmental (information on house 295 characteristics and on its immediate surrounding) as well as geographical data (soil type, watercourse nearby, vegetation index, rainfall, etc) were collected all along the follow-up. 
demonstrated in two studies on the impact of placental malaria on infant susceptibility $(34,35)$. This risk turned out to have a highly significant effect on the recurrence of events in the present study ( $\mathrm{p}<1 \times 10^{-8}$ and $\mathrm{p}<1 \times 10^{-6}$ for discovery and replication cohort respectively).

We find strong support for the involvement of protein tyrosine phosphatase (PTP) receptors. Highly suggestive association peaks were observed for PTPRM with RMM and association peaks observed for PTPRT replicated for both phenotypes. Interestingly, both genes are paralogues and in each signal a deleterious coding SNP which could be the causal SNP is identified. Although the replication of PTPRT was weak in the second cohort ( $\mathrm{p}=0.01)$, this can be explained by the fact that our replication cohort is relatively small in size, that infants in this cohort were not actively followed during the first year of life, which limited our analysis to the 12-24 months period. However, assessment of incidence rate of mild malaria attacks in function of groups of different level of exposure, showed very consistent results among exposure groups and cohorts which is an additional argument for a true association. PTPRT and PTPRM are proteins belonging to the PTP superfamily, which regulates diverse signaling pathways by catalyzing the removal of a phosphate group from specific signaling proteins. PTPRT and PTPRM encodes two PTP receptors of the same sub-family type IIb (36). These transmembrane proteins have an extracellular domain involved in cell-cell aggregation and a phosphatase domain which allows intra-cellular signaling. Interestingly PTPRT protein directly interacts with STAT3 as shown by the STRING interaction Network (Figure 4 B) (37) and is the only interactor for which a high confidence ( $>0.70)$ is reported in the STRING database. The role of PTPRT in regulating STAT3 pathways has been demonstrated, with STAT3 identified as a direct substrate of PTPRT $(38,39)$. STAT3 is a signal transducer and activator of transcription which behaves similarly to $\mathrm{NF}-\kappa \mathrm{B}$, the role of which in malaria infection is well-established. Moreover, STAT3 has been reported as associated with cerebral malaria severity in experimental studies (40,41). Liu et al. have demonstrated using murine models that 323 STAT3 is activated by $P$. berghei infection. The STAT3 pathway is thus very likely to be involved in the first stages of malaria infection and represents a potential drug target. 
325 Other genes replicated with $\mathrm{p}<0.05$ in the validation cohort. MYLK4 belongs to the family of

326 myosin ligth chain kinases (MYLKs) that catalyse myosin interaction with actin filaments. Since

327 members of MYLKs family play an essential role in the organization of the actin/myosin

328 cytoskeleton, and in cell motility (42), MYLK4 may play a role in RBC membrane structure. At

329 10q26.3 locus, replication is observed for a SNP located in KNDC1, but two eQTL associations

330 identified VENTX as the most probable effector for this association signal. VENTX is a homeobox

331 transcriptional factor that controls proliferation and differentiation of hematopoietic and immune

332 cells $(43-45)$. Wu et al. works $(44,45)$ have shown that VENTX is a key regulator of macrophage

333 terminal differentiation and of dendritic cells differentiation and maturation. VENTX is up-regulated

334 during monocyte-to-macrophage differentiation and overexpression of VENTX accelerates the

335 differentiation towards dendritic cells. Conversely, VENTX deficiency impairs dendritic cells

336 differentiation and affects various aspects of macrophage differentiation and function, including a

337 downregulation of multiple membrane receptors critical for innate and adaptive immunity. Both

338 macrophages and dendritic cells are innate immune cells derived from monocytes that play an

339 essential role in the response to malaria infections (Chua et al., 2013). Allele at risk of mild malaria

340 attacks in our data was associated with a lower expression of VENTX, which would negatively

341 impact the macrophages and/or dendritic cells response to malaria infection in the most susceptible

342 children. Finally, a high deleterious missense SNP in UROC1 (Urocanate Hydratase 1), a gene

343 expressed in the liver (GTEX RNA-seq), is also associated. Urocanate Hydratase or urocanase is an

344 enzyme that catabolizes urocanic acid to 4-imidazolone-5-propionic acid in liver. Urocanic acid is

345 found in the skin and the sweat of humans, and has been shown to protect the skin from ultra violet

346 radiation. It has also been demonstrated to be a major chemo-attractant for a skin-penetrating

347 parasitic nematode (46). Thus, urocanic acid and urocanase could be hypothesized to play a role in

348 the attractiveness of humans to mosquitoes.

349 Several strong association signals in the discovery cohort were not replicated in the validation

350 cohort. Given the limits of the replication cohort mentioned above, we think that the most 
351 promising ones are still worth consideration and functional annotation and gene mapping at these

352 loci were performed with FUMA. ACER3 identified at 11q13.5 locus appears of particular

353 relevance. It is associated both with a protection against asymptomatic and symptomatic malaria

354 infections. ACER3 is an alkaline ceramidase, involved in the degradation of ceramides into sphingosine-1-phosphatase (S1P). Ceramides have an anti-plasmodium action $(47,48)$, which is inhibited by S1P; and it has been hypothesized that antimalarial drug artemisinin and mefloquine have an antiparasitic action through the activation of sphingomyelinase producing ceramide (49). In our data allele at risk is associated with a decreased ACER3 expression, thus indicating a lower ceramidase activity in the children with higher recurrence of malaria infections. This is not in accordance with the anti-plasmodium action of ceramides as mentioned above as a lower ACER3 expression would be rather associated with a higher availability of ceramides. However ACER3 has been also involved in the activation of innate immune in mice and this could also to explain the role of ACER3 observed in malaria protection (50).

364 A second gene of interest highlighted by FUMA in malaria infections analyses is PRKN (or PARK2) coding for the parkin RBR E3 Ubiquitin Protein Ligase. Mutations in this gene are known to cause Parkinson disease and this gene has also been involved in innate immune response with a role in ubiquitin-mediated autophagy of intracellular pathogens, specifically M.tuberculosis (51).

368 Finally, a strong association signal in CSMD1 gene on chromosome 8 was evidenced in mild malaria attacks analysis. No functional evidence was found by FUMA for this association signal; however, an association signal in CSMD1 was recently found in a GWAS of severe malaria in 371 Tanzania (20); it is a transmembrane protein, assumed to be a tumor suppressor gene (52), and is 372 also associated with phenotypes as diverse as blood pressure (53) and schizophrenia (54) but there 373 is as of yet no clear scenario to explain its potential role in malaria.

374 We also examined association results for the most associated candidate variants with malariarelated phenotypes in the literature (Table 3). There was no evidence of replication for any variant, except rs40401 located in IL3 $(\mathrm{p}=0.01$ and $\mathrm{p}=0.02$ for malaria attacks and malaria infections, 
respectively). The fact that our study target population differs substantially from those used in most published studies on mild malaria may partly explain the limited evidence of replication observed.

379 HBB was not significantly associated with non-severe malaria in our sample. There are accumulating evidence for an age-related protective effect of HbS (55-57) with an acquired mechanism of protection from both asymptomatic and symptomatic $P$. falciparum infections in children. Our results are in line with two of these studies $(55,56)$ which did not detect a protective effect before the age of two.

Our study has identified several genes whose biological function is relevant to malaria physiopathology and which could play a role in the control of malaria infection. Naturally, future studies are required to further validate these findings. The difficulty of setting up such longitudinal field studies have indeed limited the number of individuals that have been included in malaria cohorts. However our results show that GWAS on non-severe malaria can successfully identify new candidate genes and inform physiological mechanisms underlying natural protection against malaria. Improving our understanding of the disease course is crucial for the development of 391 effective control measures.

\section{Materials and Methods}

\section{Study population - discovery cohort}

The discovery cohort was composed of 525 infants followed-up from birth until 18 months (58).

395 This study took place from June 2007 to January 2010 in 9 villages of Tori-bossito district located $39640 \mathrm{~km}$ North-East of Cotonou. Southern Benin is characterised by a subtropical climate, with 2 397 rainy seasons (a long rainy season from April to July and a short one in August and September). 398 Clinical incidence of malaria mainly due to $P$. falciparum (97\%) was estimated to be 1.5 (95\%CI 1.2-1.9) malaria episodes per child (0-5 years) per year in this area (59) and entomological 400 inoculation rate on average 15.5 infective bites per human per year in studied villages (33). 
401 The design and the flow-chart of the study have been published elsewhere (58). Briefly, 656 infants

402 were included at birth and followed with a close parasitological and clinical survey until the age of

40318 months. During the entire follow-up, infants were weekly visited at home by a nurse of the

404 program and temperature was systematically controlled. In case of axillary temperature higher or

405 equal to $37^{\circ} 5$ (or a history of fever in the preceding 24 hours), child was referred to health center

406 for medical screening where both a TBS and a rapid diagnostic test (RDT) were performed. Once a

407 month a systematic TBS was performed to detect asymptomatic parasitemia. At any time in case of

408 suspicious fever or clinical signs, related or not to malaria, mothers were invited to bring their

409 infants to the health center where the same protocol (temperature, TBS and RDT) was applied.

410 Symptomatic malaria infection was treated with artemether-lumefantrine combination therapy as

411 recommended by the National Malaria Control Program. A total of 10589 TBS were performed

412 along the follow-up which were read by two independent technicians.

413 To assess the risk of exposure to Anopheles bites, environmental (information on house

414 characteristics and on its immediate surrounding) and geographical data (satellite images, soil type,

415 watercourse nearby, vegetation index, rainfall, etc) were recorded. Throughout the study, every six

416 weeks, human landing catches were performed in several points of the villages to evaluate spatial

417 and temporal variations of Anopheles density. Altogether these data allowed modeling, for each

418 child included in the follow-up, an individual risk of exposure by means of a space- and time-

419 dependent variable (33).

\section{Study population - replication cohort}

421 The replication cohort was composed of 250 infants who were part of a mother-child study

422 conducted from 2009 to 2013 in the district of Allada, a southern semi-rural area located 55

423 kilometres north of Cotonou and $20 \mathrm{~km}$ from the first study site (60). Infants were born to mothers

424 who participated in a multi-country clinical trial for the prevention of malaria in pregnancy

425 (MiPPAD trial, “Malaria in Pregnancy Preventive Alternative Drugs, NCT00811421,(61)). The first 
400 newborns from MiPPAD participants (62) were enrolled in the present study. In brief, 400

Clinical malaria infections were treated as recommended by the National Malaria Control Program.

438 Environmental and geographical data were collected as well, to estimate risk of exposure for each of malaria follow-up protocols in the first year lead to the detection of a lower number of malaria infections, ii) environmental and entomological data needed to estimate the risk of exposure were

\section{Ethics Approval}

449 For the two cohort studies, both oral and written communications were provided to parent's children 
451 signed by parents who agreed to participate. The protocols of these studies were approved by both

452 the Beninese Ethical Committee of the Faculté des Sciences de la Santé (FSS) and the IRD 453 Consultative Committee on Professional Conduct and Ethics (CCDE).

\section{Genotyping and data quality control}

455 Genotyping was conducted at the Centre National de Recherche en Génomique Humaine (CNRGH, 456 CEA, Evry, France). Before genotyping, a quality control was systematically performed on each DNA sample. All samples were quantified by fluorescence, in duplicate, using the Quant-It kits (Thermo Fischer Scientific). The lowest values systematically underwent a second measurement before any sample was excluded. The quality of material was estimated using about $10 \%$ of the total samples received (selected randomly throughout the collection) by performing: i) a quality check by migration on a $1 \%$ agarose gel to ensure the samples were not degraded, ii) a standard PCR amplification reaction on the samples to ensure that the genomic DNA was free of PCR inhibitors, iii) a PCR test to verify the gender of the individual (63). All samples with concentrations below 20 ng/ $\mu \mathrm{L}$, or a major quality problem (degradation and/or amplification problems) were systematically excluded from the study.

After quality control, DNA samples were aliquoted in 96-well plates (JANUS liquid handling robot, Perkin Elmer) for genotyping; sample tracking was ensured by a systematic barcode scanning for each sample. Two DNA positive controls were systematically inserted in a random fashion into the plates. Genotyping was performed on Illumina HumanOmni5-4v1 chips, on a high throughput Illumina automated platform, in accordance with the standard automated protocol of Illumina ${ }^{\circledR}$

471 'Infinium HD Assay (Illumina ${ }^{\circledR}$, San Diego, USA).The PCR amplification of the genomic DNA 472 was performed in a dedicated ( «pre-PCR ») laboratory. Fragmentation and hybridization of the 473 DNA on the chips were performed in a dedicated («post-PCR ») laboratory. Several quality controls were systematically included during the process, such as visual inspection of the DNA pellets after precipitation, visual inspection of the deposited cocktail of reagents for hybridization, 
systematic verification of the temperature of the heating block during the extension and imaging steps. Reading of the chips was performed on iScan+ scanners (Illumina ${ }^{\circledR}$, San Diego,

Standard control steps and criteria (64) in GWAS were then applied to data. For DNA samples, individuals with discordant genotypic and reported sex were removed $(n=5)$; heterozygosity versus sample call rate were examined, leading to removal of one clear outlier; all remaining samples had a call rate $>0.97$ (mean $=0.998)$ and were kept for analysis. A genetic relationship matrix $(\mathrm{GRM})$

observed in both cohorts which was expected as most of the recruitment was made in rural villages.

One pair of individuals was removed because of an unexpectedly high kinship coefficient $(\Phi=$ identify and remove SNPs with poor reproducibility (4986 SNPs showing at least one discordance). After these quality control steps, the total sample contained 2,363,703 markers for 775 children.

\section{Population stratification}

A principal component analysis (PCA) was performed to evaluate potential population stratification in our samples. The analysis were performed on 624 pairwise unrelated individuals $(\Phi<0.05)$, after filtering on allele frequency (MAF $>0.05)$ and LD thinning $\left(\mathrm{r}^{2}<0.2\right)$. The 151 remaining individuals were then projected onto the principal components. 
PCA was performed including West African populations of the KGP. Genetic data were merged on common positions, filtered and thinned as for the first PCA. Only 100 unrelated individuals from each of the two study cohorts were selected, to give them the same weight as KGP samples in PCA. The remaining individuals were projected on the factorial plan thus obtained.

\section{Imputation}

Imputation of the two cohorts together was performed on the Michigan Imputation Server (66) . The reference allele was homogenized to the reference genome by converting the genotypes to the positive strand when necessary; duplicated SNPs, indels, monomorphic SNPs, and SNPs with inconsistent reference allele with the reference genome were removed. The resulting genotype data containing 2,539,302 SNPs were uploaded to the Michigan Imputation Server. A last QC step was performed on the server by comparing allele frequencies observed in the uploaded data and in the African reference panel from 1000 Genomes v5(67) , and flipping strand for variants showing significant difference in allele frequency ( $\chi 2$ statistics greater than 300 ). After this step, there was a high matched between genotype data and the reference panel (S5 Fig).

515 Genotype data were finally imputed using the minimac3 algorithm provided by the Michigan

516 Imputation Server. We selected SHAPEIT v2 for prephasing $(68,69)$ and all haplotypes from 1000

517 Genomes v5 (67) as the reference panel. We filtered out imputed SNPs with a squared correlation

$518\left(\mathrm{R}^{2}\right)$ between input genotypes and expected continuous dosages below 0.8 , or with a MAF below 5190.01 , which yielded an imputed genotype data set of 15,566,900 variants.

\section{Adjusting on relevant epidemiological and environmental factors}

521 Association studies were performed with RMM and RMI. A mild malaria attack was defined as a positive RDT or TBS along with fever (axillary temperature $\geq 37.5^{\circ} \mathrm{C}$ ) or a history of fever in the preceding 24 hours. Each mild malaria attack was recorded with its precise date of event and children were considered not to be at risk of malaria within the 14 days after receiving an anti- 
525 malarial treatment. Malaria infections included both symptomatic and asymptomatic infections. A

526 positive malaria diagnostic TBS without any fever or history of fever in the preceding 24 hours was

527 considered as an asymptomatic infection if the child did not experience a clinical malaria in the

528 following three days.

529 The risk of infection greatly varies over the year depending on the season and malaria transmission

530 level. This variation can be accounted for using the time dependent risk of exposure which was

531 defined for each infant in a previous work (Cottrell et al. 2012). To do so, we used a Cox model for 532 recurrent events, with a rate of events at time $t$ (Therneau et Grambsch 2000)

$$
\lambda(t)=\lambda_{0}(t) e^{X_{t} \beta+Z b}
$$

where $X_{t}$ is a design matric for (possibly time dependent) covariates with a fixed effect, $Z$ is a matrix of indicator variables designed for including a vector of random individual effects $b$ with variance proportional to the GRM $2 \Phi$, allowing to account for cryptic relatedness and population structure. The matrix $X_{t}$ includes covariates (sex, birthweights, risk of exposure at time $t$, etc). This model has the advantage of both taking into account incomplete follow-up and all the malaria infections, while incorporating time-dependent susceptibility variables.

To fit this model, the follow-up was divided in month intervals following scheduled visit. For each event we considered the risk of exposure estimated at the previous scheduled visit. A stepwise backward strategy was used to select relevant covariates. Factors considered were individual factors (sex, birth weight), health center, factors related to malaria during pregnancy (placental malaria, infection during pregnancy - replication cohort only-, intake of intermittent preventive treatment -

545 discovery cohort only- , arm of clinical trials - replication cohort only-), parity (primigravida vs multigravida), maternal anemia at delivery, use of bednet during the follow-up (categorical variable based on mother declaration during the follow-up), risk of exposure and transmission season (a time-dependent variable in four categories, both follow-up spanning over 3 years: dry season, rainy 
550 socio-economic factors (mother educational level, marital status). The final model included only

551 covariates significant at $p \leq 0.05)$.

\section{Genome-wide association analyses}

553 It would have been appealing to use the Cox model (1) for the genome-wide analysis, including the

554 genotype of each SNP to be tested for association in the covariates matrix $X_{t}$. However the

555 computational burden inherent to this modeling (with a non-sparse GRM 2Ф) makes it

556 inappropriate for the large number of tests in GWAS.

557 We thus used the Best Linear Unbiased Predictor (BLUP) $\hat{b}$ of $b$ obtained from the fitted model

558 (1), including all covariates selected by the stepwise backward procedure. This value $\hat{b}$ is an

559 “individual frailty”, corresponding to individual effects which are not explained by the

560 epidemiological covariates. To investigate genetic factors involved in these individual effects, they

561 were analyzed using a linear mixed model $\hat{b} \sim \operatorname{MVN}\left(G \gamma, 2 \tau \Phi+\sigma^{2} I d\right)$ where $G$ is the vector of

562 genotypes at the SNP to be tested, coded as 0,1 or 2 according the number of alternate alleles,

563 which corresponds to an additive model on the log hazard-ratio scale.

564 This analysis was performed for each genotyped SNP with a MAF $>0.01$, as well as with dosages

565 of imputed SNPs. The GRM matrix $2 \Phi$ used was computed as described in the quality control

566 section. The quantile-quantile plot of the $p$-values was visually inspected and the genomic inflation

567 factor $\lambda$ (ratio of the median of the observed distribution of the test statistic to the expected median)

568 was calculated to verify the absence of inflation of test statistics due to relatedness and population

569 structure.

570 SNPs that showed an association at $p \leq 10^{-5}$ in the discovery cohort were selected for replication

571 where the same two steps approach was applied. Gene-based annotations given by ANNOVAR (71)

572 and CADD score (25) were added for these variants in supplementary tables. All highly suggestive

573 signals (around the significant threshold of $p \leq 5 \times 10^{-8}$ in the discovery cohort, or which 
replicated at $p \leq 0.05$ ) were re-analysed with the mixed Cox model of equation (1) above. For

575 these signals we additionally performed a meta-analysis combining the results obtained by mixed 576 Cox models, using classical method implemented in METAL software (72).

577 All statistical analyses were done using R software (73), with the package gaston (74) for data 578 quality control and linear mixed models, and the package coxme (23) for the random effects Cox 579 model. Manhattan plots were obtained with the package qqman (75), and regional linkage 580 disequilibrium plot for association signals with Locuszoom Standalone v1.4 (76).

\section{Gene mapping and biological prioritization}

Loci that showed evidence of association at $p \leq 10^{-5}$ with one of the two phenotypes in the discovery cohort, were mapped to gene using the FUMA web plateform (FUMAGWAS v1.3.3;

http://fuma.ctglab.nl/) (27), designed for post-processing of GWAS results and prioritizing of genes. FUMA annotates candidate SNPs in genomic risk loci and subsequently mappes them to prioritized genes based on (i) physical position mapping on the genome, (ii) expression quantitative trait loci (eQTL) mapping and (iii) 3D chromatin interactions (chromatin interaction mapping). It incorporates the most recent bioinformatics databases, such as Combined Annotation Dependent Depletion score (CADD score) $(25,26)$, regulatory elements in the intergenic region of the genome (RegulomeDB) (28), Genotype-Tissue Expression (GTEx) and 3D chromatin interactions from HIC experiments (29).

We considered as genomic risk loci, loci with evidence of replication (table 1) and regions with at least 3 SNPs below the $p$-value threshold of $10^{-5}$. In the initial step, SNPs in LD $\left(r^{2}>0.01\right.$, estimated from AFR reference panel of KGP phase 3) in a $250 \mathrm{~Kb}$ windows were considered as belonging to the same risk locus. Thereafter, a set of candidate SNPs were selected, based on LD $\left(r^{2}>0.6\right)$ with the lead SNP (defined as SNP with the lowest p-value) or with replicating SNPs from our dataset and from AFR population data of KGP phase 3. 
to define deleterious SNPs was a CADD score $>12.37$ which indicates potential pathogenicity or a

600 RegulomeDB score $\leq 2$ which indicates that the SNP likely lies in a functional location (categories

6011 and 2 of Regulome classification identified as “likely to affect binding”). EQTL mapping was

602 performed using eQTL data from tissue types relevant for non-severe malaria. Search for eQTL

603 associations were carried out in Blood eQTL (77), BIOS QTL (30), eQTLGen, three databases of e604 QTL associations identified in blood, and in the following tissue types of GTEx v7 database: whole 605 blood, cells-transformed fibroblast, cells-EBV-transformed lymphocytes, liver, skin (sun exposed or 606 not) and spleen. Chromatin interaction mapping was performed from Hi-C experiments data of five tissues/cell types identified as relevant for non-severe malaria: liver, spleen GM12878, IMR90 and trophoblast-like-cell. Hi-C experiments identified chromatin interaction between small

chromosomal regions two by two. In this analysis a candidate SNP is mapped to a gene if a significant interaction is observed between a first region containing the candidate SNP and the 611 second one where the gene is located. FDR was used to correct for multiple testing (FDR $<0.05$ for eQTL association and FDR $<1 \times 10^{-6}$ for chromatin interaction, the default values in FUMA).

\section{Candidate associations}

We report associations observed in the discovery cohort for genes ( $\pm 200 \mathrm{~kb}$ 3’ and 5’) showing the greatest evidence of association with malaria in the literature. Genes previously associated with mild malaria phenotype were selected from the review by Marquet (7): $\mathrm{HbS}$ and $\mathrm{HbC}$ alleles of $H B B, I L 3$ from the 5q31-q33 region, TNF, NCR3 from 6p21-p23 region, and IL10. Association was not assessed for the HP gene and apha-thalasemia condition, because the structural variants involved are not directly accessible through high density genotyping array (78). We also checked significance for genes previously associated with severe malaria that were confirmed by recent

621 GWA and multi-center studies: ABO, ATP2B4, and FREM3/GYP locus (17-19,22).

\section{Acknowledgements}

623 This research is a collaboration between the CEA/ Jacob/CNRGH and the IRD/UMR216. We wish 
to thanks the collaborators of the CERPAGE who participated actively in the longitudinal followups. Longitudinal follow-ups were funded by the ANR grants (ANR-SEST 2006 040-01 and ANRPRSP 2010 012-001); the Ministère de la Recherche et des Technologies, France (REFS Nu2006-

22) and the IRD. We made use of data previously generated in the MiPPAD study (EDCTPcomputing and storage resources.

\section{References}

1. World Health Organization. WHO | World malaria report 2018. 2018.

2. Bhatt S, Weiss DJ, Cameron E, Bisanzio D, Mappin B, Dalrymple U, et al. The effect of malaria control on Plasmodium falciparum in Africa between 2000 and 2015. Nature. 2015 Oct 8;526(7572):207-11.

3. Verra F, Mangano VD, Modiano D. Genetics of susceptibility to Plasmodium falciparum: from classical malaria resistance genes towards genome-wide association studies. Parasite Immunol. 2009 May;31(5):234-53.

4. Kwiatkowski DP. How malaria has affected the human genome and what human genetics can teach us about malaria. Am J Hum Genet. 2005 Aug;77(2):171-92.

5. Mackinnon MJ, Mwangi TW, Snow RW, Marsh K, Williams TN. Heritability of malaria in Africa. PLoS Med. 2005 Dec;2(12):e340.

6. Driss A, Hibbert JM, Wilson NO, Iqbal SA, Adamkiewicz TV, Stiles JK. Genetic polymorphisms linked to susceptibility to malaria. Malar J. 2011 Sep 19;10:271.

7. Marquet S. Overview of human genetic susceptibility to malaria: From parasitemia control to severe disease. Infect Genet Evol J Mol Epidemiol Evol Genet Infect Dis. 2017 Jun 1;

8. Garcia A, Marquet S, Bucheton B, Hillaire D, Cot M, Fievet N, et al. Linkage analysis of blood Plasmodium falciparum levels: interest of the 5q31-q33 chromosome region. Am J Trop Med Hyg. 1998 Jun;58(6):705-9.

9. Rihet P, Traoré Y, Abel L, Aucan C, Traoré-Leroux T, Fumoux F. Malaria in humans: Plasmodium falciparum blood infection levels are linked to chromosome 5q31-q33. Am J Hum Genet. 1998 Aug;63(2):498-505.

10. Flori L, Kumulungui B, Aucan C, Esnault C, Traoré AS, Fumoux F, et al. Linkage and association between Plasmodium falciparum blood infection levels and chromosome 5q31q33. Genes Immun. 2003 Jun;4(4):265-8.

11. Sakuntabhai A, Ndiaye R, Casadémont I, Peerapittayamongkol C, Peerapittayamonkol C, Rogier C, et al. Genetic determination and linkage mapping of Plasmodium falciparum malaria 
related traits in Senegal. PloS One. 2008;3(4):e2000.

12. Jepson A, Sisay-Joof F, Banya W, Hassan-King M, Frodsham A, Bennett S, et al. Genetic linkage of mild malaria to the major histocompatibility complex in Gambian children: study of affected sibling pairs. BMJ. 1997 Jul 12;315(7100):96-7.

13. Brisebarre A, Kumulungui B, Sawadogo S, Atkinson A, Garnier S, Fumoux F, et al. A genome scan for Plasmodium falciparum malaria identifies quantitative trait loci on chromosomes 5q31, 6p21.3, 17p12, and 19p13. Malar J. 2014 May 28;13:198.

14. Timmann C, Evans JA, König IR, Kleensang A, Rüschendorf F, Lenzen J, et al. Genome-wide linkage analysis of malaria infection intensity and mild disease. PLoS Genet. 2007 Mar 23;3(3):e48.

15. Milet J, Nuel G, Watier L, Courtin D, Slaoui Y, Senghor P, et al. Genome wide linkage study, using a 250K SNP map, of Plasmodium falciparum infection and mild malaria attack in a Senegalese population. PloS One. 2010;5(7):e11616.

16. Baaklini S, Afridi S, Nguyen TN, Koukouikila-Koussounda F, Ndounga M, Imbert J, et al. Beyond genome-wide scan: Association of a cis-regulatory NCR3 variant with mild malaria in a population living in the Republic of Congo. PloS One. 2017;12(11):e0187818.

17. Timmann C, Thye T, Vens M, Evans J, May J, Ehmen C, et al. Genome-wide association study indicates two novel resistance loci for severe malaria. Nature. 2012 Sep 20;489(7416):443-6.

18. Band G, Le QS, Jostins L, Pirinen M, Kivinen K, Jallow M, et al. Imputation-based metaanalysis of severe malaria in three African populations. PLoS Genet. 2013 May;9(5):e1003509.

19. Malaria Genomic Epidemiology Network, Band G, Rockett KA, Spencer CCA, Kwiatkowski DP. A novel locus of resistance to severe malaria in a region of ancient balancing selection. Nature. 2015 Oct 8;526(7572):253-7.

20. Ravenhall M, Campino S, Sepúlveda N, Manjurano A, Nadjm B, Mtove G, et al. Novel genetic polymorphisms associated with severe malaria and under selective pressure in Northeastern Tanzania. PLoS Genet. 2018 Jan;14(1):e1007172.

21. Malaria Genomic Epidemiology Network, Malaria Genomic Epidemiology Network. Reappraisal of known malaria resistance loci in a large multicenter study. Nat Genet. 2014 Nov;46(11):1197-204.

22. Leffler EM, Band G, Busby GBJ, Kivinen K, Le QS, Clarke GM, et al. Resistance to malaria through structural variation of red blood cell invasion receptors. Science. 2017 16;356(6343).

23. Terry M. Therneau. coxme: Mixed Effects Cox Models. [Internet]. Available from: https://CRAN.R-project.org/package=coxme

24. Adzhubei IA, Schmidt S, Peshkin L, Ramensky VE, Gerasimova A, Bork P, et al. A method and server for predicting damaging missense mutations. Nat Methods. 2010 Apr;7(4):248-9.

25. Kircher M, Witten DM, Jain P, O’Roak BJ, Cooper GM, Shendure J. A general framework for estimating the relative pathogenicity of human genetic variants. Nat Genet. 2014

Mar;46(3):310-5. 
26. Rentzsch P, Witten D, Cooper GM, Shendure J, Kircher M. CADD: predicting the deleteriousness of variants throughout the human genome. Nucleic Acids Res. 2019 Jan 8;47(D1):D886-94.

27. Watanabe K, Taskesen E, van Bochoven A, Posthuma D. Functional mapping and annotation of genetic associations with FUMA. Nat Commun. 2017 28;8(1):1826.

28. Boyle AP, Hong EL, Hariharan M, Cheng Y, Schaub MA, Kasowski M, et al. Annotation of functional variation in personal genomes using RegulomeDB. Genome Res. 2012 Sep;22(9):1790-7.

29. Schmitt AD, Hu M, Jung I, Xu Z, Qiu Y, Tan CL, et al. A Compendium of Chromatin Contact Maps Reveals Spatially Active Regions in the Human Genome. Cell Rep. 2016 15;17(8):2042-59.

30. Zhernakova DV, Deelen P, Vermaat M, van Iterson M, van Galen M, Arindrarto W, et al. Identification of context-dependent expression quantitative trait loci in whole blood. Nat Genet. 2017;49(1):139-45.

31. Greenwood BM. The microepidemiology of malaria and its importance to malaria control. Trans R Soc Trop Med Hyg. 1989;83 Suppl:25-9.

32. Bousema T, Drakeley C, Gesase S, Hashim R, Magesa S, Mosha F, et al. Identification of hot spots of malaria transmission for targeted malaria control. J Infect Dis. 2010 Jun 1;201(11):1764-74.

33. Cottrell G, Kouwaye B, Pierrat C, le Port A, Bouraïma A, Fonton N, et al. Modeling the influence of local environmental factors on malaria transmission in Benin and its implications for cohort study. PloS One. 2012;7(1):e28812.

34. Le Port A, Cottrell G, Chandre F, Cot M, Massougbodji A, Garcia A. Importance of adequate local spatiotemporal transmission measures in malaria cohort studies: application to the relation between placental malaria and first malaria infection in infants. Am J Epidemiol. 2013 Jul 1;178(1):136-43.

35. Bouaziz O, Courtin D, Cottrell G, Milet J, Nuel G, Garcia A. Is Placental Malaria a Long-term Risk Factor for Mild Malaria Attack in Infancy? Revisiting a Paradigm. Clin Infect Dis Off Publ Infect Dis Soc Am. 2018 Mar 5;66(6):930-5.

36. Nikolaienko RM, Agyekum B, Bouyain S. Receptor protein tyrosine phosphatases and cancer. Cell Adhes Migr. 2012 Jul 1;6(4):356-64.

37. Szklarczyk D, Morris JH, Cook H, Kuhn M, Wyder S, Simonovic M, et al. The STRING database in 2017: quality-controlled protein-protein association networks, made broadly accessible. Nucleic Acids Res. 2017 Jan 4;45(D1):D362-8.

38. Zhang X, Guo A, Yu J, Possemato A, Chen Y, Zheng W, et al. Identification of STAT3 as a substrate of receptor protein tyrosine phosphatase T. Proc Natl Acad Sci U S A. 2007 Mar 6;104(10):4060-4.

39. Peyser ND, Freilino M, Wang L, Zeng Y, Li H, Johnson DE, et al. Frequent promoter hypermethylation of PTPRT increases STAT3 activation and sensitivity to STAT3 inhibition in head and neck cancer. Oncogene. 2016 Mar 3;35(9):1163-9. 
40. Liu M, Amodu AS, Pitts S, Patrickson J, Hibbert JM, Battle M, et al. Heme mediated STAT3 activation in severe malaria. PloS One. 2012;7(3):e34280.

41. Liu M, Solomon W, Cespedes JC, Wilson NO, Ford B, Stiles JK. Neuregulin-1 attenuates experimental cerebral malaria (ECM) pathogenesis by regulating ErbB4/AKT/STAT3 signaling. J Neuroinflammation. 2018 Apr 10;15(1):104.

42. Tan I, Leung T. Myosin light chain kinases: division of work in cell migration. Cell Adhes Migr. 2009 Sep;3(3):256-8.

43. Gao H, Wu X, Sun Y, Zhou S, Silberstein LE, Zhu Z. Suppression of homeobox transcription factor VentX promotes expansion of human hematopoietic stem/multipotent progenitor cells. J Biol Chem. 2012 Aug 24;287(35):29979-87.

44. Wu X, Gao H, Ke W, Giese RW, Zhu Z. The homeobox transcription factor VentX controls human macrophage terminal differentiation and proinflammatory activation. J Clin Invest. 2011 Jul;121(7):2599-613.

45. Wu X, Gao H, Bleday R, Zhu Z. Homeobox transcription factor VentX regulates differentiation and maturation of human dendritic cells. J Biol Chem. 2014 May 23;289(21):14633-43.

46. Safer D, Brenes M, Dunipace S, Schad G. Urocanic acid is a major chemoattractant for the skin-penetrating parasitic nematode Strongyloides stercoralis. Proc Natl Acad Sci U S A. 2007 Jan 30;104(5):1627-30.

47. Labaied M, Dagan A, Dellinger M, Gèze M, Egée S, Thomas SL, et al. Anti-Plasmodium activity of ceramide analogs. Malar J. 2004 Dec 10;3:49.

48. Heung LJ, Luberto C, Del Poeta M. Role of Sphingolipids in Microbial Pathogenesis. Infect Immun. 2006 Jan;74(1):28-39.

49. Pankova-Kholmyansky I, Dagan A, Gold D, Zaslavsky Z, Skutelsky E, Gatt S, et al. Ceramide mediates growth inhibition of the Plasmodium falciparum parasite. Cell Mol Life Sci CMLS. 2003 Mar;60(3):577-87.

50. Wang K, Xu R, Snider AJ, Schrandt J, Li Y, Bialkowska AB, et al. Alkaline ceramidase 3 deficiency aggravates colitis and colitis-associated tumorigenesis in mice by hyperactivating the innate immune system. Cell Death Dis. 2016 Mar 3;7:e2124.

51. Manzanillo PS, Ayres JS, Watson RO, Collins AC, Souza G, Rae CS, et al. PARKIN ubiquitin ligase mediates resistance to intracellular pathogens. Nature. 2013 Sep 26;501(7468):512-6.

52. Sun PC, Uppaluri R, Schmidt AP, Pashia ME, Quant EC, Sunwoo JB, et al. Transcript map of the 8p23 putative tumor suppressor region. Genomics. 2001 Jul;75(1-3):17-25.

53. Hong K-W, Go MJ, Jin H-S, Lim J-E, Lee J-Y, Han BG, et al. Genetic variations in ATP2B1, CSK, ARSG and CSMD1 loci are related to blood pressure and/or hypertension in two Korean cohorts. J Hum Hypertens. 2010 Jun;24(6):367-72.

54. Håvik B, Le Hellard S, Rietschel M, Lybæk H, Djurovic S, Mattheisen M, et al. The complement control-related genes CSMD1 and CSMD2 associate to schizophrenia. Biol Psychiatry. 2011 Jul 1;70(1):35-42. 
55. Williams TN, Mwangi TW, Roberts DJ, Alexander ND, Weatherall DJ, Wambua S, et al. An immune basis for malaria protection by the sickle cell trait. PLoS Med. 2005 May;2(5):e128.

56. Gong L, Maiteki-Sebuguzi C, Rosenthal PJ, Hubbard AE, Drakeley CJ, Dorsey G, et al. Evidence for both innate and acquired mechanisms of protection from Plasmodium falciparum in children with sickle cell trait. Blood. 2012 Apr 19;119(16):3808-14.

57. Lopera-Mesa TM, Doumbia S, Konaté D, Anderson JM, Doumbouya M, Keita AS, et al. Effect of red blood cell variants on childhood malaria in Mali: a prospective cohort study. Lancet Haematol. 2015 Apr;2(4):e140-149.

58. Le Port A, Cottrell G, Martin-Prevel Y, Migot-Nabias F, Cot M, Garcia A. First malaria infections in a cohort of infants in Benin: biological, environmental and genetic determinants. Description of the study site, population methods and preliminary results. BMJ Open. 2012;2(2):e000342.

59. Damien GB, Djènontin A, Rogier C, Corbel V, Bangana SB, Chandre F, et al. Malaria infection and disease in an area with pyrethroid-resistant vectors in southern Benin. Malar J. 2010 Dec 31;9:380.

60. d'Almeida TC, Sadissou I, Milet J, Cottrell G, Mondière A, Avokpaho E, et al. Soluble human leukocyte antigen -G during pregnancy and infancy in Benin: Mother/child resemblance and association with the risk of malaria infection and low birth weight. PloS One. 2017;12(2):e0171117.

61. González R, Mombo-Ngoma G, Ouédraogo S, Kakolwa MA, Abdulla S, Accrombessi M, et al. Intermittent preventive treatment of malaria in pregnancy with mefloquine in HIV-negative women: a multicentre randomized controlled trial. PLoS Med. 2014 Sep;11(9):e1001733.

62. Accrombessi M, Ouédraogo S, Agbota GC, Gonzalez R, Massougbodji A, Menéndez C, et al. Malaria in Pregnancy Is a Predictor of Infant Haemoglobin Concentrations during the First Year of Life in Benin, West Africa. PloS One. 2015;10(6):e0129510.

63. Wilson JF, Erlandsson R. Sexing of human and other primate DNA. Biol Chem. 1998 Oct;379(10):1287-8.

64. Anderson CA, Pettersson FH, Clarke GM, Cardon LR, Morris AP, Zondervan KT. Data quality control in genetic case-control association studies. Nat Protoc. 2010 Sep;5(9):1564-73.

65. Yang J, Lee SH, Goddard ME, Visscher PM. GCTA: a tool for genome-wide complex trait analysis. Am J Hum Genet. 2011 Jan 7;88(1):76-82.

66. Das S, Forer L, Schönherr S, Sidore C, Locke AE, Kwong A, et al. Next-generation genotype imputation service and methods. Nat Genet. 2016;48(10):1284-7.

67. Sudmant PH, Rausch T, Gardner EJ, Handsaker RE, Abyzov A, Huddleston J, et al. An integrated map of structural variation in 2,504 human genomes. Nature. 2015 Oct 1;526(7571):75-81.

68. Delaneau O, Marchini J, Zagury J-F. A linear complexity phasing method for thousands of genomes. Nat Methods. 2011 Dec 4;9(2):179-81.

69. Delaneau O, Zagury J-F, Marchini J. Improved whole-chromosome phasing for disease and population genetic studies. Nat Methods. 2013 Jan;10(1):5-6. 
70. Therneau TM, Grambsch PM. Modeling Survival Data: Extending the Cox Model [Internet]. New York: Springer-Verlag; 2000 [cited 2018 Apr 26]. (Statistics for Biology and Health). Available from: //www.springer.com/us/book/9780387987842

71. Wang K, Li M, Hakonarson H. ANNOVAR: functional annotation of genetic variants from high-throughput sequencing data. Nucleic Acids Res. 2010 Sep;38(16):e164.

72. Willer CJ, Li Y. METAL: fast and efficient meta-analysis of genomewide association scans. Bioinforma Oxf Engl. 2010 Sep 1;26(17):2190-1.

73. R Core Team. R: A language and environment for statistical computing. R Foundation for Statistical Computing [Internet]. R Foundation for Statistical Computing; 2017. Available from: https://www.R-project.org

74. Hervé Perdry \& Claire Dandine-Roulland. gaston: Genetic Data Handling (QC, GRM, LD, PCA) \& Linear Mixed Models. 2018.

75. Stephen Turner. qqman: Q-Q and Manhattan Plots for GWAS Data. [Internet]. 2014. Available from: https://CRAN.R-project.org/package=qqman

76. Pruim RJ, Welch RP, Sanna S, Teslovich TM, Chines PS, Gliedt TP, et al. LocusZoom: regional visualization of genome-wide association scan results. Bioinformatics. 2010 Sep 15;26(18):2336-7.

77. Westra H-J, Peters MJ, Esko T, Yaghootkar H, Schurmann C, Kettunen J, et al. Systematic identification of trans eQTLs as putative drivers of known disease associations. Nat Genet. 2013 Oct;45(10):1238-43.

78. Higgs DR. The Molecular Basis of $\alpha$-Thalassemia. Cold Spring Harb Perspect Med. 2013 Jan;3(1). 